OPEN ACCESS

Edited by:

Anja Joachim,

University of Veterinary Medicine

Vienna, Austria

Reviewed by:

Hans-Peter Fuehrer,

University of Veterinary Medicine,

Vienna, Austria

Christina Strube,

University of Veterinary Medicine

Hannover, Germany

${ }^{*}$ Correspondence:

Josué Martínez-de la Puente jmp@ebd.csic.es

Rubén Bueno-Mari

rbueno@/okimica.es

Specialty section:

This article was submitted to

Parasitology,

a section of the journal

Frontiers in Veterinary Science

Received: 13 April 2020

Accepted: 23 June 2020

Published: 21 August 2020

Citation:

Martínez-de la Puente J, Soriguer $R$, Senar JC, Figuerola J, Bueno-Mari R and Montalvo T (2020) Mosquitoes in an Urban Zoo: Identification of Blood

Meals, Flight Distances of Engorged

Females, and Avian Malaria Infections.

Front. Vet. Sci. 7:460

doi: 10.3389/fvets.2020.00460

\section{Mosquitoes in an Urban Zoo: Identification of Blood Meals, Flight Distances of Engorged Females, and Avian Malaria Infections}

\author{
Josué Martínez-de la Puente ${ }^{1,2 *}$, Ramón Soriguer ${ }^{1,2}$, Juan Carlos Senar ${ }^{3}$, \\ Jordi Figuerola ${ }^{1,2}$, Rubén Bueno-Mari ${ }^{4 *}$ and Tomás Montalvo ${ }^{2,5}$ \\ ${ }^{1}$ Estación Biológica de Doñana (EBD-CSIC), Sevilla, Spain, ${ }^{2}$ CIBER Epidemiología y Salud Pública (CIBERESP), Madrid, \\ Spain, ${ }^{3}$ Evolutionary and Behavioural Ecology Research Unit, Museu de Ciències Naturals de Barcelona, Barcelona, Spain, \\ ${ }^{4}$ Laboratorios Lokímica, Departamento de Investigación y Desarrollo (I+D), Valencia, Spain, ${ }^{5}$ Agencia de Salud Pública de \\ Barcelona, Consorci Sanitari de Barcelona, Barcelona, Spain
}

Zoological gardens are home to a large number of vertebrate species and as such are suitable sites for both mosquito breeding and maintenance. They are excellent places for entomological studies of mosquito phenology, diversity, and blood-feeding patterns, as well as for xenomonitoring. During 2016, we sampled mosquitoes in Barcelona Zoo and used molecular methods to determine their blood-feeding patterns and the prevalence and diversity of avian malaria parasites. We also estimated the flight distance of engorged mosquitoes in the area. Overall, 1,384 adult Culex pipiens s.l., Culiseta Iongiareolata, and Aedes albopictus were captured. Birds dominated the diet of Cx. pipiens s.l. $(n=87)$ and Cs. longiareolata $(n=6)$, while humans were the only blood-meal source of $A e$. albopictus $(n=3)$. Mosquitoes had a mean flight distance of $95.67 \mathrm{~m}$ after feeding on blood (range 38.71-168.51 m). Blood parasites were detected in the abdomen of 13 engorged Cx. pipiens s.l., eight of which had fed on magpies. Four Plasmodium lineages and a single lineage of the malaria-like parasite Haemoproteus were identified. These results suggest that $C x$. pipiens s.l. is involved in the local transmission of avian Plasmodium, which potentially affects the circulation of parasites between and within wildlife and enclosed animals. Vigilance regarding possible mosquito breeding sites in this zoo is thus recommended.

\section{Keywords: Aedes albopictus, avian Plasmodium, Culex pipiens, malaria, vectors}

\section{INTRODUCTION}

Mosquitoes transmit a diversity of vector-borne pathogens affecting humans, livestock, and wildlife $(1,2)$. In addition to native species, invasive mosquitoes such as alien Aedes mosquitoes are involved in the circulation of both imported and locally circulating pathogens (3). This is the case of the invasive Asian tiger mosquito Aedes albopictus, which is associated with the local transmission of pathogens such as filarioid worms (e.g., Dirofilaria spp.), protozoa (e.g., avian malaria parasites), and viruses (e.g., Dengue virus) (4-6). This species has a broad global distribution and is present in countries outside its native range in America, Europe, Oceania, and Africa (7). In Spain, Ae. albopictus was first recorded in 2004 in Catalonia and since then has progressively colonized different parts of this region (8). 
Zoos and wildlife parks with non-autochthonous and stabled fauna are excellent sites for studying the ecology and epidemiology of vector-borne pathogens, as studies of sand flies (9, 10), biting midges Culicoides (11), and mosquitoes (12-15) have previously shown. Given that captive animals are housed in known locations, the flight distances of captured insect vectors containing blood from these animals can be accurately estimated (10). In addition, these areas are frequented by human visitors and there are often both freely moving animals and animals maintained in captivity, all of which are potentially exposed to the transmission of various pathogens (15). This is especially the case of avian malaria parasites of the genus Plasmodium, a group of haemosporidians that naturally circulates between birds and mosquitoes (16) that can severely affect the health and survival of birds in zoos and recovery centers (17-22). However, in spite of their veterinary importance, the transmission dynamics of mosquito-borne avian Plasmodium in these particular ecosystems are still poorly understood [e.g., $(12,15,23,24)]$.

Here, we sampled mosquitoes in Barcelona Zoo, a site with a great diversity of mosquito and bird species, many of which are infected by avian malaria parasites (25). We employed a comprehensive approach based on the analysis of mosquito blood-feeding patterns, xenomonitoring (defined as the identification of pathogens in mosquito vectors), and flight distances of engorged females. We used molecular methods to identify the blood-meal sources of both native and alien engorged mosquitoes and to screen for the presence of avian Plasmodium in the abdomens of engorged mosquitoes to assess contact rates between potential vectors and parasites in the area.

\section{METHODS}

\section{Study Area, Mosquito Sampling, and Species Identification}

Mosquitoes were collected in June-November 2016 in Barcelona Zoo using both passive and active trapping techniques (Figure 1). BG-Sentinel traps (Biogents $\mathrm{GmbH}$, Regensburg, Germany) were installed for $24 \mathrm{~h}$ in three different sites in the zoo, namely, the aviary, the farm, and the terrarium. These sites were selected according to criteria related to host proximity, distance between traps, and cover and protection for the traps. Every 2 weeks, mosquitoes were sampled using BGSentinel traps baited with $\mathrm{CO}_{2}$ during $24 \mathrm{~h}$. In addition, we used entomological aspirators (Improved Prokopack Aspirator, Mod. 1419, John W. Hock Company, FL, USA, and CDC Backpack Aspirator Mod. 2846, BioQuip, CA, USA) to collect mosquitoes resting on vegetation, bins, and animal cages. Aspirations were performed in six pre-established sites distributed throughout the whole zoo (13.5 ha) for $5 \mathrm{~min}$ in each area to standardize active adult mosquito sampling and increase the overall number of blood-fed mosquitoes collected. Additionally, 10 standard ovitraps $(350 \mathrm{ml}$ water capacity) were installed and monitored weekly to obtain information on mosquito phenology. Larval samplings were conducted occasionally to check for the presence/absence of other species that had not been collected by the above-described methodologies. Mosquitoes were identified using morphological keys (26); females containing any remains of blood meals in their abdomens were stored individually at $-80^{\circ} \mathrm{C}$ until subsequent molecular analyses.

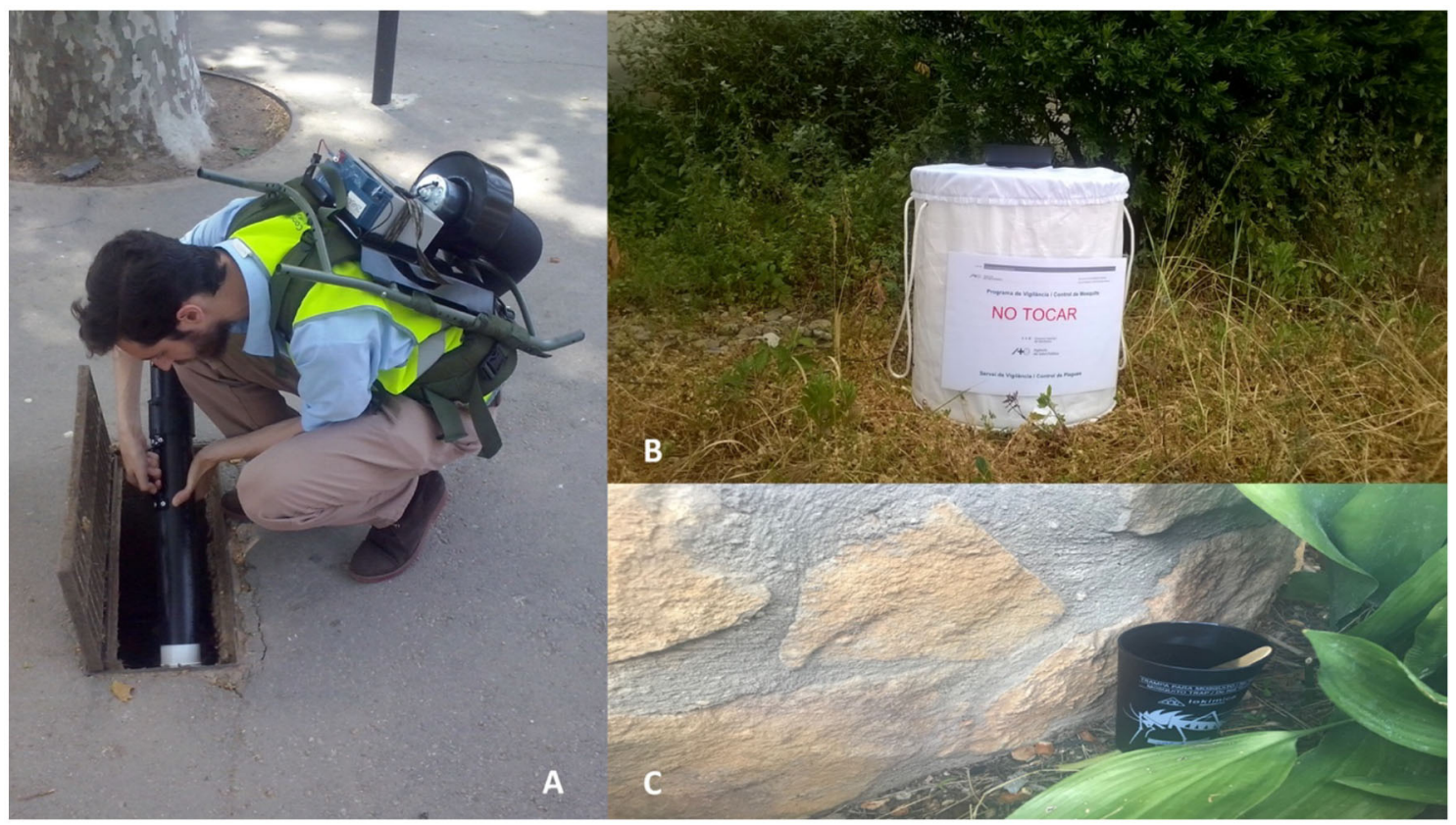

FIGURE 1 | Sampling methods used in this study including direct aspiration in mosquito resting areas (A), BG-Sentinel traps (B), and ovitraps (C). 


\section{Molecular Identification of Blood-Meal Sources and Parasites in Engorged Mosquitoes}

The abdomen of each engorged mosquito was separated from the head-thorax using sterile tips on Petri dishes. Genomic DNA was isolated from these abdomens using Maxwell ${ }^{\circledR} 16$ LEV System Research Kit (Promega, Madison, WI). The protocol described by Alcaide et al. (27) involving the amplification of the barcoding region of the cytochrome oxidase subunit 1 (COI) gene was used to identify the blood-meal origin of mosquitoes [see also (28) for the adaptation of the protocol for Ae. albopictus]. The presence of avian Plasmodium/Haemoproteus parasites was screened for following Hellgren et al. (29). Amplified fragments were sequenced using the Macrogen Inc. facilities (Amsterdam, The Netherlands), and the resulting sequences were edited using Sequencher ${ }^{\mathrm{TM}}$ v4.9 (Gene Codes Corp., (C) 1991-2009, Ann Arbor, MI 48108, USA). Blood-meal sources were identified by comparing sequences obtained with those deposited in public-access databases (GenBank DNA, National Center for Biotechnology Information Blast and the Barcode of Life Data Systems), bearing in mind the species potentially present in the study area. Parasite lineages and, whenever possible, their respective morpho-species were identified by Blast comparison with sequences in GenBank and/or MalAvi (30).

\section{Analyses of Flight Distances of Blood-Fed Female Mosquitoes}

We estimated the minimum flight distances of mosquitoes using data concerning where mosquitoes were captured and the locations of caged host species (Figure 2). Mosquito trapping areas and enclosures within the zoo of identified vertebrate host species were georeferenced. Only vertebrate species in enclosures in the zoo were considered in the analyses. Freely moving hosts (e.g., humans) were excluded from the analyses. Based on host identification, distances between the centroids of the areas enclosing animals and mosquito sampling points (BG-Sentinel stations or entomological aspiration points) were measured to calculate flight distances. Analyses were carried out with QGIS 3.12 (QGIS Development Team. http://qgis.osgeo.org).

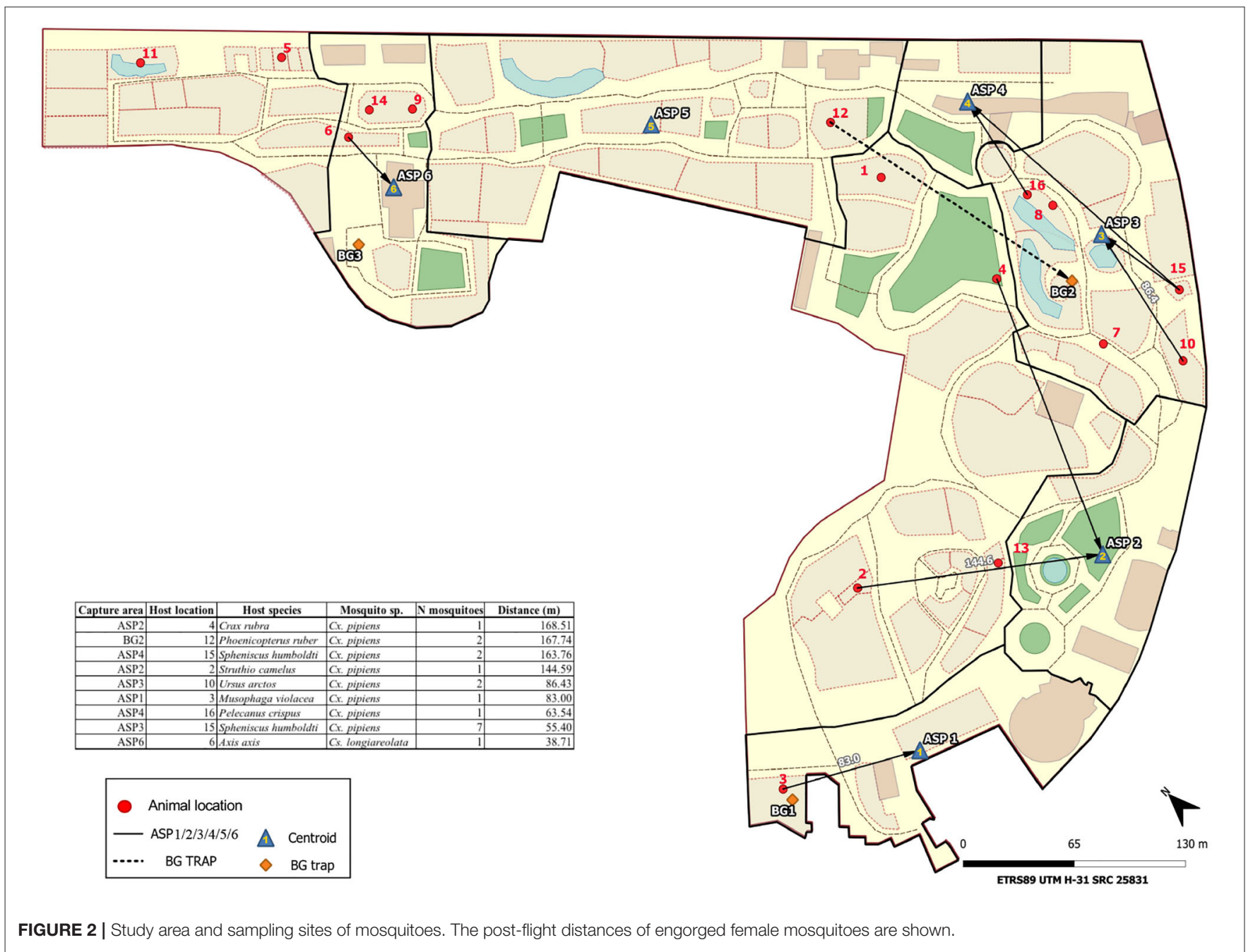




\section{RESULTS}

We trapped 1,384 adult Culex pipiens s.l. ( $n=733 ; 52.96 \%)$, Ae. albopictus $(n=490 ; 35.40 \%)$, and Culiseta longiareolata $(n=161 ; 11.63 \%)$. In total, $63.29 \%(n=876)$ of these mosquitoes were collected using entomological aspirators and the remaining $36.71 \%(n=508)$ using BG-Sentinel traps. Catches of $C x$. pipiens s.l. peaked in June-July, while those of Ae. albopictus adults peaked in September-October; these results were supported by both direct collection by entomological aspirators and the BGSentinel traps. In addition, we collected 9,658 Ae. albopictus eggs, with a maximum in July-October and a peak in August (3,965 eggs).

In all, 137 (9.90\%) mosquitoes had some blood remains in their abdomens, of which the vertebrate hosts of 86 (62.32\%) were successfully identified (birds and mammals) (Table 1). Birds dominated the diet of $C x$. pipiens s.l. $(92.21 \% ; n=77)$. The three identified Ae. albopictus blood meals came from humans, while the six blood meals from Cs. longiareolata corresponded to birds. Post-blood-feeding flight distances were calculated for the 18 mosquitoes that had fed on enclosed animals (17 Cx. pipiens s.l. and a single Cs. longiareolata) (Figure 2). For Cx. pipiens s.l., the mean post-blood feeding flight distance was $99.02 \mathrm{~m}$, with a maximum of $168.51 \mathrm{~m}$. The only distance measured for Cs. longiareolata was 38.71 meters. Overall, 13 (9.49\%) out of the 137 engorged mosquitoes analyzed were positive for the presence of parasites (Table 1). All the parasite lineages found in this study coincided with Plasmodium lineages: Delurb4 $(n=1)$, Delurb5 $(n=3)$, Syat05 $(n=1$, corresponding to $P$. vaughani), SGS1 ( $n=1$, corresponding to $P$. relictum), and the Haemoproteus lineage hCIRCUM04 ( $n=5$, also called BLUTI9). Sequences corresponding to each of the five lineages identified in this study were deposited in GenBank (MT568928-MT568932). In addition, one Plasmodium parasite and one Haemoproteus parasite were detected in mosquitoes, although the low quality of these sequences did not allow us to accurately identify their parasite lineages. Twelve out of the 13 positive mosquitoes corresponded to $C x$. pipiens s.l. A single Cs. longiareolata was positive for Haemoproteus lineage hCIRCUM04.

\section{DISCUSSION}

Infections by Plasmodium parasites and related haemosporidians are commonly found in birds in zoos (31) and can lead to illness and/or lethal diseases (20-22). These cases usually occur in immunologically naïve species such as penguins originating from areas where the parasites circulating in zoos are not present. These animals are frequently bitten by competent vectors of avian malaria parasites, especially of the genus Culex (18), and largely suffer the costs of infections $(17,19)$. Interestingly, we found that a number of $C x$. pipiens s.l. had fed on Humboldt penguins (Spheniscus humboldti), including mosquitoes with P. relictum. Different treatments and prophylactic protocols are applied to penguins to minimize the parasite-induced illness (18), as is also common practice in the study area.

Our results regarding the blood-feeding pattern of mosquitoes support the hypothesis that $C x$. pipiens s.l. females feed mainly on
TABLE 1 | Vertebrate hosts of mosquitoes trapped in the zoological garden of Barcelona during 2016.

\begin{tabular}{|c|c|c|c|c|}
\hline Host & $\begin{array}{c}\text { Ae. } \\
\text { albopictus }\end{array}$ & $\begin{array}{c}\text { Cs. } \\
\text { longiareolata }\end{array}$ & $\begin{array}{c}\text { Cx. } \\
\text { pipiens }\end{array}$ & Parasites \\
\hline \multicolumn{5}{|l|}{ Birds } \\
\hline Ardea cinerea & & & 12 & \\
\hline Ardea sp. & & 1 & & \\
\hline Bubulcus ibis & & 1 & 18 & \\
\hline Columba livia & & & 1 & Haemoproteus sp. (1) \\
\hline Corvus monedula & & & 1 & \\
\hline Egretta garzetta/sp. & & & 4 & \\
\hline Crax rubra & & & 1 & \\
\hline Musophaga violacea & & & 1 & \\
\hline Myiopsitta monachus & & & 3 & \\
\hline Passer domesticus & & 1 & & \\
\hline Pavo sp. & & & 2 & \\
\hline Pelecanus sp. & & & 1 & \\
\hline Phoenicopterus ruber & & & 2 & P. vaughani SYAT05 (1) \\
\hline Pica pica & & 2 & 8 & $\begin{array}{l}\text { Haemoproteus } \\
\text { hCIRCUM04 (3), } \\
\text { Plasmodium Delurb5 } \\
\text { (3), Plasmodium } \\
\text { Delurb4 (1), } \\
\text { Plasmodium sp. (1) }\end{array}$ \\
\hline Spheniscus humboldti & & & 9 & P. relictum SGS1 (1) \\
\hline Streptopelia decaocto & & & 7 & $\begin{array}{l}\text { Haemoproteus } \\
\text { hCIRCUM04 (1) }\end{array}$ \\
\hline Struthio camelus & & & 1 & \\
\hline \multicolumn{5}{|l|}{ Mammals } \\
\hline Axis axis & & 1 & & \\
\hline Canis lupus & & & 1 & \\
\hline Homo sapiens & 3 & & 3 & \\
\hline Ursus arctos & & & 2 & \\
\hline
\end{tabular}

The parasite lineages identified in mosquitoes fed on each animal are shown. One mosquito with an unidentified blood meal was positive for Haemoproteus (hCIRCUM04).

birds $(6,28,32)$. Despite the extremely low sample size, we also found support for the ornithophilic behavior of Cs. longiareolata; however, Ae. albopictus fed exclusively on humans. This latter species is a nuisance for visitors, especially in summer when it is commonest. These results provide valuable information regarding the blood-feeding sources of these species, which is especially relevant in the case of Ae. albopictus as this subject has only to date been investigated at a handful of studies in Europe $(6,25,28,33)$. Identification of the blood-meal sources of mosquitoes in zoos allows researchers to investigate additional aspects of vector ecology such as the flight distances of engorged females. Greenberg et al. (13) recorded an average distance of $106.7 \mathrm{~m}$ for mosquitoes of the genus Aedes (Ae. vexans), Culex (Cx. Quinquefasciatus, and Cx. tarsalis), and Culiseta (Cs. inornata) in USA. Similar values were reported by Tuten et al. (34) in zoos from USA, where Anopheles and Culex mosquitoes flew a mean distance of $94.1 \mathrm{~m}$ after feeding. Ejiri et al. (12) found that the post-blood-meal flight distance of Cx. pipiens pallens lay in the range $10-350 \mathrm{~m}$ in Japan. Heym et al. (14) estimated the mean post-blood-meal flight distance of Cx. pipiens (biotype pipiens) in two zoos in Germany as 132.2 
and $362.3 \mathrm{~m}$. In this study in a Mediterranean area, engorged Cx. pipiens s.l. and Cs. longiareolata were captured in average at $95.67 \mathrm{~m}$ from their hosts, with a maximum of $168.51 \mathrm{~m}$ for $C x$. pipiens s.l. It is worth highlighting that only a single female of Cs. longiareolata was found to have blood from zoo animals and so further studies targeting this species are still required. We were not able to calculate the flight distances of Ae. albopictus due to that fact that in this study it fed exclusively on humans.

Our results suggest that encounters between infected birds and competent mosquito vectors frequently occur in the area, which probably facilitates the transmission of avian Plasmodium within this zoo $(12,15,23,24)$. Previous studies in the Iberian Peninsula have identified avian Plasmodium in mosquitoes of the genera Culex and Aedes $(25,32,35,36)$, and of them, $C x$. pipiens probably plays a key role in the transmission of avian Plasmodium due to its wide distribution (1), competence for the transmission of different avian Plasmodium lineages/species $(16,37)$, and its ornithophilic behavior $(6,32)$. A previous study in the same area also identified the presence of avian Plasmodium in Cx. pipiens s.l. pools (25). Nevertheless, the molecular identification of parasite DNA in mosquitoes does not necessarily imply vector competence (38) and so we were not able to assess the competence of these mosquitoes for avian malaria transmission. This is the case, above all, of Haemoproteus parasites, a parasite genus transmitted by Culicoides spp. and louse flies (16), that cannot be effectively transmitted by mosquitoes.

\section{CONCLUSION}

Barcelona Zoo is a suitable site for the development and maintenance of both native and alien species of mosquitoes. Based on their blood-feeding patterns, Cx. pipiens s.l. probably play a role in the local transmission and spread of mosquitoborne pathogens. This ornithophilic mosquito is a well-known vector of avian Plasmodium parasites. Thus, Cx. pipiens s.l. and the parasites transmitted may have an impact on the health of caged birds and so very likely represent a veterinary concern. In addition, magpies are common hosts of mosquitoes in the area and it is likely that they act as Plasmodium spp. reservoirs since

\section{REFERENCES}

1. Farajollahi A, Fonseca DM, Kramer LD, Marm KA. "Bird biting" mosquitoes and human disease: a review of the role of Culex pipiens complex mosquitoes in epidemiology. Infect Genet Evol. (2011) 11:157785. doi: 10.1016/j.meegid.2011.08.013

2. Folly AJ, Dorey-Robinson D, Hernández-Triana LM, Phipps LP, Johnson N. Emerging threats to animals in the United Kingdom by arthropodborne diseases. Front Vet Sci. (2020) 7:20. doi: 10.3389/fvets.2020. 00020

3. Medlock JM, Hansford KM, Schaffner F, Versteirt V, Hendrickx G, Zeller $\mathrm{H}$, et al. A review of the invasive mosquitoes in Europe: ecology, public health risks, control options. Vector Borne Zoonotic Dis. (2012) 12:43547. doi: 10.1089/vbz.2011.0814 high parasite prevalence has been found in the mosquitoes that feed on this bird species.

\section{DATA AVAILABILITY STATEMENT}

All data analysed during this study are included in this published article, any further information is available from the corresponding author on reasonable request.

\section{ETHICS STATEMENT}

Ethical approval was not required for this study according to national/local legislation because mosquitoes are not protected by any law. Written informed consent was obtained from the individual for the publication of any potentially identifiable images or data included in this article.

\section{AUTHOR CONTRIBUTIONS}

JM, JF, RB-M, and TM designed the study. JS, RB-M, and TM performed the fieldwork. JM, RS, JF, RB-M, and TM analyzed the samples and data. RS, JF, RB-M, and TM contributed with reagents. JM drafted the first version of the manuscript. All authors read and approved this manuscript.

\section{FUNDING}

This study was funded by projects CGL2015-65055-P, CGL-201679568-C3-3-P, and PGC2018-095704-B-100 from the Spanish Ministry of Science and Innovation and with the support of the Fundación Barcelona Zoo and Barcelona city Council.

\section{ACKNOWLEDGMENTS}

We thank Isabel Martín and Laura Gómez for their help in the laboratory and Helena Navalpotro for her help collecting mosquitoes. Lokímica contributed assistant workers and logistic support, in particular, Juan López, and Cristina Sesé. The work of the JM and JF was done within the framework of AIM-COST Action CA17108. Two reviewers provided valuable comments on an earlier version of this manuscript. 
8. Collantes F, Delacour S, Alarcón-Elbal PM, Ruiz-Arrondo I, Delgado JA, Torrell-Sorio A, et al. Review of ten-years presence of Aedes albopictus in Spain 2004-2014: known distribution and public health concerns. Parasit Vectors. (2015) 8:655. doi: 10.1186/s13071-015-1262-y

9. Muñoz C, Martínez-de la Puente J, Figuerola J, Pérez-Cutillas P, Navarro $\mathrm{R}$, Ortuño $\mathrm{M}$, et al. Molecular xenomonitoring E, and host identification of Leishmania sand fly vectors in a Mediterranean periurban wildlife park. Transbound Emerg Dis. (2019) 66:2546-61. doi: 10.1111/tbed.13319

10. Pérez-Cutillas P, Muñoz C, Martínez-de la Puente J, Figuerola J, Navarro R, Ortuño $\mathrm{M}$, et al. A spatial ecology study in a high-diversity host community to understand blood-feeding behaviour in Phlebotomus sandfly vectors of Leishmania. Med Vet Entomol. (2020) 34:164-174. doi: 10.1111/mve.12427

11. England ME, Pearce-Kelly P, Brugman VA, King S, Gubbins S, Sach F, et al. Culicoides species composition and molecular identification of host blood meals at two zoos in the UK. Parasit Vectors. (2020) 13:139. doi: 10.1186/s13071-020-04018-0

12. Ejiri H, Sato Y, Kim KS, Hara T, Tsuda Y, Imura T, et al. Entomological study on transmission of avian malaria parasites in a zoological garden in Japan: blood meal identification and detection of avian malaria parasite DNA from bloodfed mosquitoes. J Med Entomol. (2011) 48:600-7. doi: 10.1603/ME10197

13. Greenberg JA, DiMenna MA, Hanelt B, Hofkin BV. Analysis of post-blood meal flight distances in mosquitoes utilizing zoo animal blood meals. $J$ Vector Ecol. (2012) 37:83-9. doi: 10.1111/j.1948-7134.2012.00203.x

14. Heym EC, Kampen H, Schäfer M, Walther D. Mosquito bloodmeal preferences in two zoological gardens in Germany. Med Vet Entomol. (2019) 33:203-12. doi: 10.1111/mve.12350

15. Heym EC, Kampen H, Krone O, Schäfer M, Werner D. Molecular detection of vector-borne pathogens from mosquitoes collected in two zoological gardens in Germany. Parasitol Res. (2019) 118:2097-105. doi: 10.1007/s00436-019-06327-5

16. Santiago-Alarcon D, Palinauskas V, Schaefer HM. Diptera vectors of avian Haemosporidian parasites: untangling parasite life cycles and their taxonomy. Biol Rev Camb Philos Soc. (2012) 87:928-64. doi: 10.1111/j.1469-185X.2012.00234.x

17. Sijbranda DC, Hunter S, Howe L, Lenting B, Argilla L, Gartrell D, et al. Cases of mortality in little penguins (Eudyptula minor) in New Zealand associated with avian malaria. N Z Vet J. (2017) 65:3327. doi: 10.1080/00480169.2017.1359124

18. Grilo ML, Vanstreels RE, Wallace R, García-Párraga D, Braga ÉM, Chitty J, et al. Malaria in penguins - current perceptions. Avian Pathol. (2016) 45:393-407. doi: 10.1080/03079457.2016.1149145

19. Vanstreels RE, Kolesnikovas CK, Sandri S, Silveira P, Belo NO, Ferreira Junior FC, et al. Outbreak of avian malaria associated to multiple species of Plasmodium in magellanic penguins undergoing rehabilitation in southern Brazil. PLoS ONE. (2014) 9:e94994. doi: 10.1371/journal.pone.0094994

20. Bueno MG, Lopez RP, de Menezes RM, Costa-Nascimento Mde J, Lima GF, Araújo RA, et al. Identification of Plasmodium relictum causing mortality in penguins (Spheniscus magellanicus) from São Paulo Zoo, Brazil. Vet Parasitol. (2010) 173:123-127. doi: 10.1016/j.vetpar.2010.06.026

21. Jia T, Huang X, Valkiunas G, Yang M, Zheng C, Pu T, et al. Malaria parasites and related Haemosporidians cause mortality in cranes: a study on the parasites diversity, prevalence and distribution in Beijing Zoo. Malar J. (2018) 17:234. doi: 10.1186/s12936-018-2385-3

22. Olias P, Wegelin M, Zenker W, Freter S, Gruber AD, Klopfleisch R, et al. Avian malaria deaths in parrots, Europe. Emerg Infect Dis. (2011) 17:9502. doi: 10.3201/eid1705.101618

23. Ejiri H, Sato Y, Sawai R, Sasaki E, Matsumoto R, Ueda M, et al. Prevalence of avian malaria parasite in mosquitoes collected at a zoological garden in Japan. Parasitol Res. (2009) 105:629-33. doi: 10.1007/s00436-009-1434-9

24. Ejiri H, Sato Y, Kim KS, Tsuda Y, Murata K, Saito K, et al. Blood meal identification and prevalence of avian malaria parasite in mosquitoes collected at Kushiro wetland, a subarctic zone of Japan. J Med Entomol. (2011) 48:9048. doi: 10.1603/ME11053
25. Martínez-de la Puente J, Díez-Fernández A, Montalvo T, Bueno-Marí $\mathrm{R}$, Pangrani Q, Soriguer RC, et al. Do invasive mosquito and bird species alter avian malaria parasite transmission? Diversity. (2020) 12:111. doi: 10.3390/d12030111

26. Schaffner E, Angel G, Geoffroy B, Hervy JP, Rhaiem A, Brunhes J. The Mosquitoes of Europe: An Identification and Training Programme. Montpellier: IRD Éditions \& EID Méditerrannée. (2001).

27. Alcaide M, Rico C, Ruiz S, Soriguer R, Muñoz J, Figuerola J, et al. Disentangling vector-borne transmission networks: a universal DNA barcoding method to identify vertebrate hosts from arthropod bloodmeals. PLoS ONE. (2009) 4:e7092. doi: 10.1371/journal.pone.0007092

28. Muñoz J, Eritja R, Alcaide M, Montalvo T, Soriguer RC, Figuerola J, et al. Host-feeding patterns of native Culex pipiens and invasive Aedes albopictus mosquitoes (Diptera: Culicidae) in urban zones from Barcelona, Spain. J Med Entomol. (2011) 48:956-60. doi: 10.1603/ME11016

29. Hellgren O, Waldenström J, Bensch S. A new PCR assay for simultaneous studies of Leucocytozoon, Plasmodium, and Haemoproteus from avian blood. J Parasitol. (2004) 90:797-802. doi: 10.1645/GE-184R1

30. Bensch S, Hellgren O, Pérez-Tris J. MalAvi: a public database of malaria parasites and related haemosporidians in avian hosts based on mitochondrial cytochrome b lineages. Mol Ecol Resour. (2009) 9:13538. doi: 10.1111/j.1755-0998.2009.02692.x

31. Chagas CRF, Valkiunas G, de Oliveira Guimarães L, Monteiro EF, Guida FJ, Simões RF, et al. Diversity and distribution of avian malaria and related haemosporidian parasites in captive birds from a Brazilian megalopolis. Malar J. (2017) 16:83. doi: 10.1186/s12936-017-1729-8

32. Martínez-de la Puente J, Ferraguti M, Ruiz S, Roiz D, Soriguer RC, Figuerola J, et al. Culex pipiens forms and urbanization: effects on blood feeding sources and transmission of avian Plasmodium. Malar J. (2016) 15:589. doi: 10.1186/s12936-016-1643-5

33. Valerio L, Marini F, Bongiorno G, Facchinelli L, Pombi M, Caputo B, et al. Host-feeding patterns of Aedes albopictus (Diptera: Culicidae) in urban and rural contexts within Rome province, Italy. Vector Borne Zoonotic Dis. (2010) 10:291-4. doi: 10.1089/vbz.2009.0007

34. Tuten HC, Bridges WC, Paul KS, Adler PH. Blood-feeding ecology of mosquitoes in zoos. Med Vet Entomol. (2012) 26:407-16. doi: 10.1111/j.1365-2915.2012.01012.x

35. Ferraguti M, Martínez-de la Puente J, Muñoz J, Roiz D, Ruiz S, Soriguer R, et al. Avian Plasmodium in Culex and Ochlerotatus mosquitoes from southern Spain: effects of season and host-feeding source on parasite dynamics. PLoS ONE. (2013) 8:e66237. doi: 10.1371/journal.pone.0066237

36. Ventim R, Ramos JA, Osório H, Lopes RJ, Pérez-Tris J, Mendes L, et al. Avian malaria infections in western European mosquitoes. Parasitol Res. (2012) 111:637-45. doi: 10.1007/s00436-012-2880-3

37. Gutiérrez-López R, Martínez-de la Puente J, Gangoso L, Soriguer R, Figuerola J. Plasmodium transmission differs between mosquito species and parasite lineages. Parasitology. (2020) 147:441-7. doi: 10.1017/S0031182020 000062

38. Valkiunas G. Haemosporidian vector research: marriage of molecular and microscopical approaches is essential. Mol Ecol. (2011) 20:30846. doi: 10.1111/j.1365-294X.2011.05187.x

Conflict of Interest: The authors declare that the research was conducted in the absence of any commercial or financial relationships that could be construed as a potential conflict of interest.

Copyright (c) 2020 Martínez-de la Puente, Soriguer, Senar, Figuerola, BuenoMari and Montalvo. This is an open-access article distributed under the terms of the Creative Commons Attribution License (CC BY). The use, distribution or reproduction in other forums is permitted, provided the original author(s) and the copyright owner(s) are credited and that the original publication in this journal is cited, in accordance with accepted academic practice. No use, distribution or reproduction is permitted which does not comply with these terms. 